\title{
Identification of pre-Catastrophic Failure Mechanisms in High Power GaN HEMT
}

\author{
Benjamin D. Huebschman ${ }^{1}$, Frank Crowne ${ }^{1}$, Ali M. Darwish ${ }^{2}$, Edward A. Viveiros ${ }^{1}$, Khamsouk Kingkeo ${ }^{1}$, Neil \\ Goldsman $^{3}$ \\ ${ }^{1}$ U.S. Army Research Laboratory, Adelphi, MD 20783 USA \\ ${ }^{2}$ Department of Electrical and Computer Engineering, University of Maryland, \\ College Park, Maryland, 20742, USA \\ ${ }^{3}$ Electronics Engineering Department, American University in Cairo, Cairo, Egypt
}

\begin{abstract}
Extended relibability measurements
The research describes an investigation into an observed phenomenon believed to be correlated with catastrophic device failure cause by the breakdown in the gate structure in high power, high frequency GaN HEMTs. Several devices that were stressed on an elevated temperature extended reliability test station showed significant changes in gate current prior to catastrophic failure. In an effort to electrically examine the devices during the breakdown process, similar devices were stressed on wafer. Detailed measurements were performed on the devices at regular intervals. On several devices, the behavior of interest was reproduced. Of the periodic measurements performed, a gate current sweep provided the greatest insight into device operation. Explanations for the observed phenomena are discussed.
\end{abstract} down.

Index Terms - Gallium nitride, HEMT, reliability, gate break

\section{INTRODUCTION}

As GaN begins to acquire for itself a secure niche as a material for high power high frequency solid state amplifiers, persistent technical challenges continue to limit the ability of this compound semiconductor to realize its full potential. The power density in high frequency GaN devices is unprecedented in solid state devices [1,2], and understanding the protracted effects of this level of power density is one of the issues facing the research community with regards to GaN. Comprehending and quantifying the trade space between performance and reliability is one of the challenges that must be solved before GaN devices can be reliably integrated into the full spectrum of the existing communication architecture. This experiment was part of a larger reliability investigation program whose goal was to determine the rate of decline in performance and the mean time to failure of the GaN HEMT fabrication process being investigated. Devices from one of the domestic GaN foundries were being investigated on an extended reliability test station. The procedure involved the commonly used Arrhenius multi-temperature reliability measurement [3, 4, 5]. Catastrophic failures were observed. These failures resulted in the complete destruction of the device making further attempts to diagnose it impossible on the failed device. A recently constructed diagnostic apparatus was available that was capable of stressing a device on wafer and at regular intervals performing tests of sufficient complexity to create a large signal model of the device [6]. In this manner, the device could be completely characterized while its performance declined. The periodic measurements provide an electrical picture of the device during the process of degradation. This facilitated further analysis into the physical cause of the device failure.

\section{SYSTEM AND MEASUREMENTS}

There were two sources of data for this research. These are the results from the initial multi-temperature reliability measurements and the diagnostic measurements.

\section{A. Reliability Measurements}

A multi- channel extended reliability test station was used to monitor the device performance of a series of fixtured AlGaN/GaN HEMTs. The devices under test (DUTs) were operated in class $\mathrm{AB}$ mode at an elevated baseplate temperature. The DUTs were driven 1.5-2 dB into compression. The input/output power, gate and drain voltages and currents, and fixture temperature were monitored throughout the test.

The failure criterion for the reliability measurement was a degradation in performance of $1 \mathrm{~dB}$ in output power; however, the dominant method of failure was not a gradual decline in performance, but was a catastrophic failure resulting in the destruction of the DUT. For a specific group of devices, the catastrophic failure was correlated with a dramatic increase in the magnitude of the gate current. A representative case of the gate current increase preceding a decline in performance and the subsequent catastrophic failure is shown in Fig. 1. In this figure, the change in gate current is observed at slightly greater than 1000 hours. The DUT fails catastrophically at before 1200 hours. 


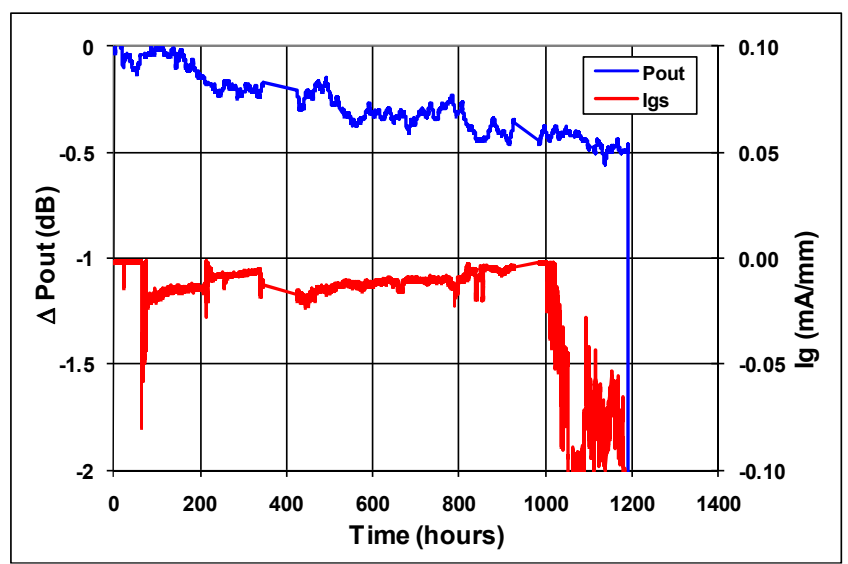

Figure 1. Fixtured reliability measurement of GaN HEMT showing output power and gate current as a function of time under stress for a device operated at a base plate temperature of $160 \mathrm{C}$.

\section{B. Diagnostic Measurements}

In order to attempt to acquire greater information on the electrical behavior of the devices being examined, several devices fabricated by the same vendor using a similar process were evaluated in a separate experiment. These devices were measured on wafer. The experiment involved stressing a device by driving it into compression in manner that was similar to the original reliability measurement. At regular intervals, the input RF power was removed from the DUT; and a series of diagnostic test were performed. These tests included S-parameter measurements and voltage-current measurements to determine the gate current and the drain current under different bias conditions. The experimental setup allowed the devices to be matched to an optimal efficiency load impedance and source impedance while being stressed. The performance of each device was recorded for a minimum of 48 hours at base plate chuck temperature of 25C, 75C, and 125C. A plot showing the output power of a device operated at $1.5 \mathrm{~dB}$ compression is plotted as a function of time while being stressed and is shown in Fig. 2. The gate current for the reliability measurement is plotted on the same graph. These data are from the last of the series of three experiments performed on the same device, and the data shown were measured while the device was being operated at $125 \mathrm{C}$. The change in gate current that was observed in the fixtured reliability measurements was again observed in the diagnostic measurements.

The interval between detailed tests was six hours. Of the series of periodic tests performed on the DUT, the results of the gate current provided the greatest insight into the possible failure mechanism. Fig. 3 shows the gate current measurements performed at periodic intervals. The complete measurement sweep and times of the measurement are displayed in Fig. 3a. The reverse bias gate behavior is shown in Fig $3 \mathrm{~b}$, and the forward bias gate behavior is shown in Fig 3c. The behavior at 125C follows two separate trends. During the first sixty hours of the experiment, the behavior followed one trend. During this period, a gradual increase in the turn-on voltage is observed in the forward bias, and there is a decrease in the leakage current when the gate is reverse biased. After the first sixty hours, there is the previously described dramatic change in the gate current. After this, the trend in gate I-V sweep changes. The turn-on voltage decreases to less than $0.5 \mathrm{~V}$ and continues to decrease as the device continues to be stressed. The reverse leakage current also increases after sixty hours and continues to increase with time.

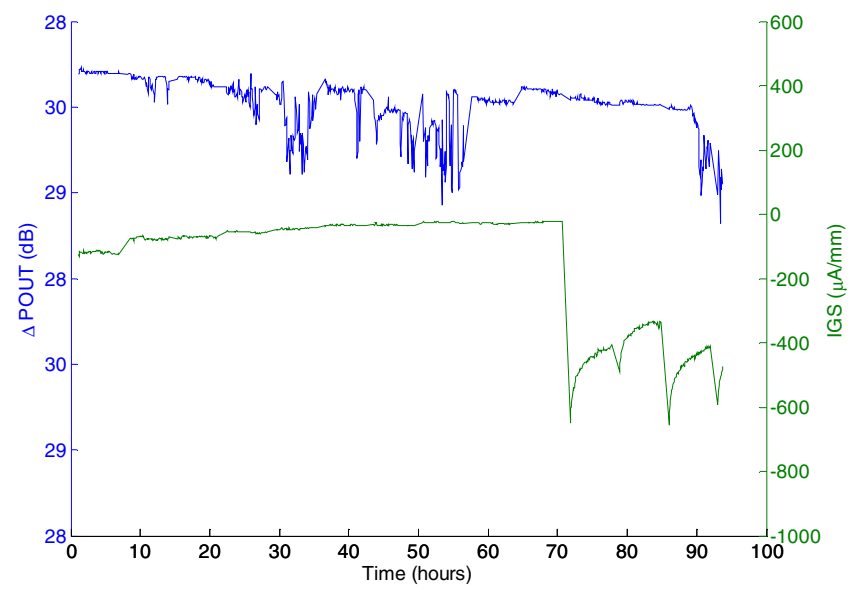

Figure 2. Stress measurement for a GaN HEMT made on wafer at 40 $\mathrm{GHz}$ with a chuck temperature of $125 \mathrm{C}$ showing output power and gate current as a function of time. The time includes only time under stress and does not include time during which diagnostic measurements were made.

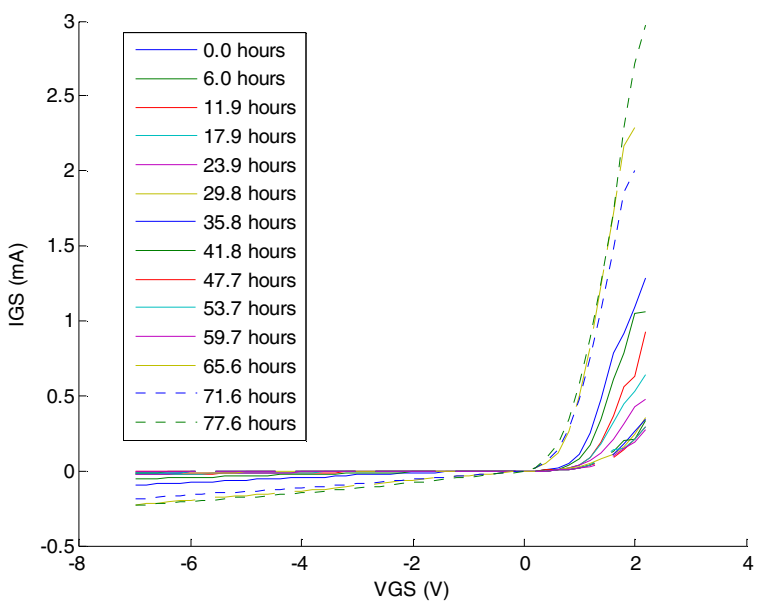

(a) 


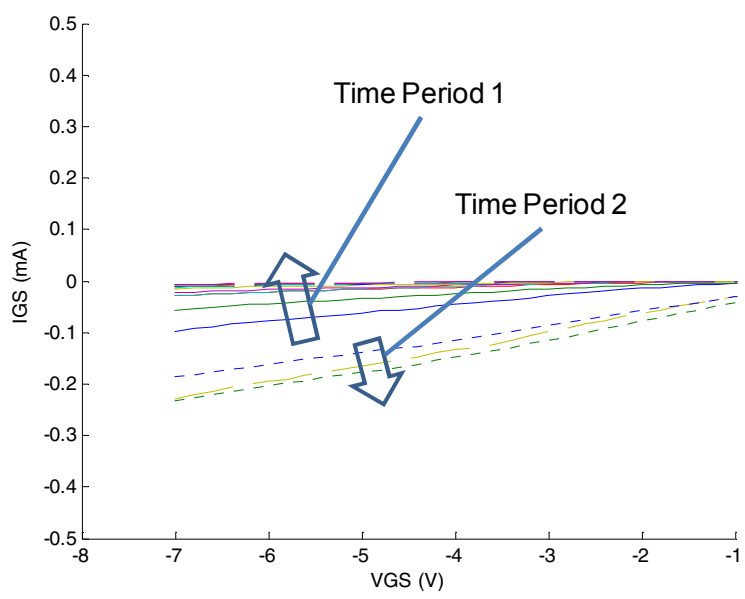

(b)

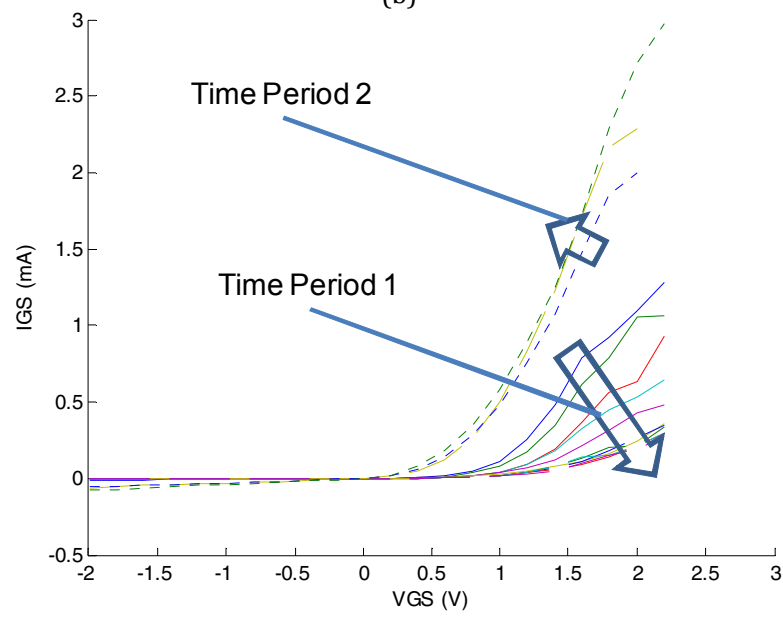

(c)

Figure 3. Periodic gate current measurements from GaN HEMT at 125 C. Showing (a) the full sweep with key and (b) trends on the the reverse bias behavior and (c) the forward bias behavior.

\section{DISCUSSION}

Under ideal operating conditions, any FET should draw essentially zero gate current under either forward or reverse gate bias. This restriction is normally implemented by placing a barrier between the channel and the gate electrode. Thus, a sudden increase in gate current suggests that a new current path has been created from the gate to the source electrode. This type of failure mode involves either:

1. Creation of a current path along the surface between the gate and source electrode, or

\section{Breach of the barrier.}

In a normal III-V HEMT, the barrier is primarily in the supply layer (e.g., the AlGaAs layer in a GaAs HEMT), and is created by (1) the heterojunction band offset and (2) the supply layer doping distribution.[7] The GaN HEMT has the additional features of (3) spontaneous polarization and
(4) piezoelectric charge at the heterojunction interface.[8] All of these charges are functions of the gate voltage, and contribute to the transfer characteristics of the FET. In addition all of them are vulnerable to modification by external probes: the heterojunction and the spontaneous polarization are sensitive to changes in the doping distribution caused by field-induced motion of the doping atoms [9], and the piezoelectric charge by relaxation of stress at the interface due to field-induced motion of dislocations [10].

Current transport through an intact barrier is primarily via tunneling, which is sensitive both to the height and the width of the barrier. In discussing barrier penetration, a useful parameter is the barrier strength $V w^{2}$, where $V$ is the barrier height and $w$ the barrier width. The effects described above can all lead to increased tunneling current via changes in the barrier strength. However, because diffusion is a slow process, it is hard to see how mechanisms (1) and (3) could produce a sudden change in the barrier characteristics. Although the effect of AlGaN donor states on HEMT electrical behavior is controversial, the published donor densities reported in the literature are large [11]; however, they lie deep in the AlGaN band gap, so much so that they resemble traps more than donors. The mechanism of dislocation motion is hard to quantify.

The reverse-bias data shown in Fig. $3 \mathrm{~b}$ for time period 1 indicate increasing suppression of the gate current for a given bias voltage, i.e., a strengthening of the barrier. This explanation must be consistent with the results for forward bias shown in Fig. 3c. Since the current flow involves tunneling through the AlGaN supply layer, it is tempting to view the gate region as if it were a nonideal Schottky barrier and argue that the same effect that creates field modifications in the reverse-bias case also modifies the tunneling barrier through which the gate current must flow.

In Fig. 4, we plot the IV characteristic of a nonideal Schottky barrier[12] with zero-turn on voltage, using the expression

$$
I=I_{S}\left(\exp \left[\frac{q V}{n k T}\right]-1\right)
$$

for several values of the nonideality. The similarity to the curves in Fig. $3 \mathrm{~b}$ is evident. Less easy to understand is the clear tendency for the diode to become more ideal with aging: although inhibition of the tunneling process will produce less current, making this a reasonable hypothesis for explaining the data, such behavior is not an obvious consequence of aging. Another possible explanation for the behavior in Period 1 is to look at the change as merely a decline in current. This could be explained by an increase in resistance at some point in the circuit path. The Period 2 data is easier to understand, as it indicates a reestablishment of the tunneling process, perhaps related to avalanche in the AlGaN layer, for which the current-voltage 
curve is much steeper than the Schottky law would predict, or to crack propagation[13]. Further study of this mechanism is needed.

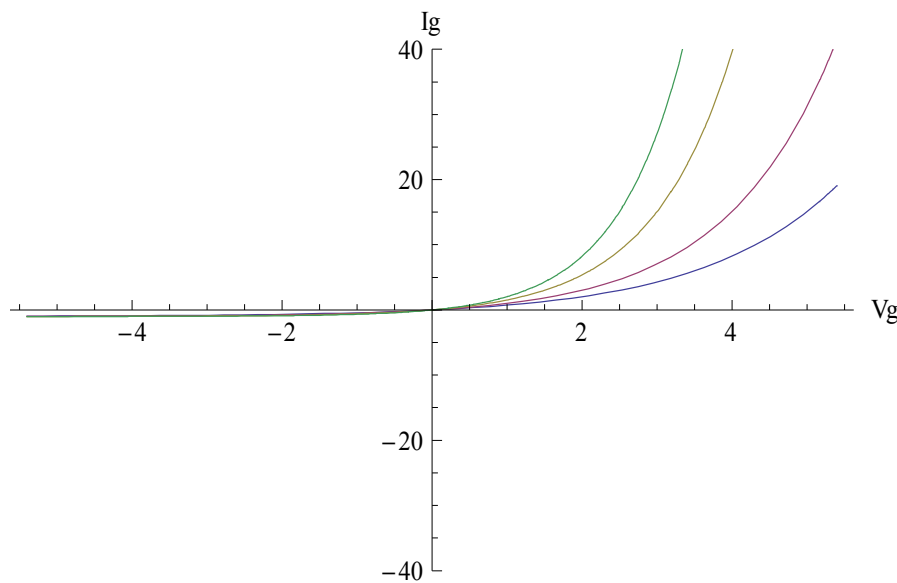

Figure. 4. Effect of nonideality on diode characteristics. Curves from lowest to highest: ideality $n=1$ (ideal diode), $n=1.25, n=1.6, n=2.5$.

\section{CONCLUSION}

The investigation of reliability can be approached from a statistical perspective that looks at the aggregate of all failure mechanism. This is useful for mature technologies with relatively long duration and gradual failure mechanisms. The alternative is to perform detailed investigation with the goal of isolating the specific failure mechanism. That was the goal in this research. We have attempted to isolate the actual failure mechanism by recording the changes to the electrical behavior of the DUT during the degradation process. In this paper we have described how an observed failure mechanism in high power GaN HEMTs served as motivation for further investigation of a more detailed nature. The phenomenon was reproduced in a controlled experiment while at the same time additional data were recorded. The precatastrophic increase in gate current was correlated with an increase in the nonideality of the gate resulting in a decrease in the gate diode turn on voltage and with an increase in the gate current.

\section{REFERENCES}

[1] T. Palacios, A. Chakraborty, S. Rajan, C. Poblenz, S. Keller, S.P. DenBaars, J. Speck, U. Mishra, "High-power AlGaN/GaN HEMTs for Ka-band applications," IEEE Electron Device Letters, volume 26, issue 11, pp. $781-783,2005$.

[2] Y.-F. Wu, A. Saxler, M. Moore, T. Wisleder, U.K. Mishra, P. Parikh, "Field-plated GaN HEMTs and amplifiers," CSIC 2005 Digest, pp. 4, Oct. 2005.

[3] S. Lee, R. Vetury, J. D. Brown, S. R. Gibbs, W. Z. Cai, J. Sun, D. S. Green, and J. B. Shealy, "Reliability assessment of AlGaN/GaN HEMT technology on $\mathrm{SiC}$ for $48 \mathrm{~V}$ applications," in Proc. IEEE Int. Reliability Physics Symp., 2008, pp. 446-449.

[4] J. A. Mitterederl, S. C. Binari, G. D. Via, I A. Roussost, J. D. Caldwell, J. P. Calamel, RF "Arrhenius life testing of X-band GaN HEMTs," in ROCS proceedings; 2008, pp. 195.

[5] R.J. Trew, D.S. Green, J.B. Shealy, "AlGaN/GaN HFET reliability," IEEE Microwave Mag., 2009, pp. 116-127.

[6] B. Huebschman, " Investigation of Reliability in Gallium Nitride High Electton Mobility Transistors using Equivalent Circuit Models," doctoral dissertation, Dept. Electrical and Computer Systems Engineering, University of Maryland, College Park, 2010.

[7] F. Stern, "Doping considerations for heterojunctions", Appl. Phys. Lett. vol. 43, 1983, pp. 974.

[8] O. Ambacher et al., "Role of spontaneous and piezoelectric polarization induced effects in group-III nitride based heterostructures and devices", Phys. Status Solidi (b), vol. 216, 1999, pp. 381.

[9] S. T. Bradley, A. P. Young, L. J. Brillson, M. J. Murphy, and W. J. Schaff, "Role of barrier and buffer layer defect states in AlGaN/GaN HEMT structures", J. of Electronic Materials, vol. 30 , 2001, pp. 123.

[10] M. Nardelli, et al., "Strain effects on the interface properties of nitride semiconductors", Phys. Rev B, vol. 55, 1997, pp. R7323.

[11] J. C. Freeman, (2003). "Basic Equations for the Modeling of Gallium Nitride (GaN) High Electron Mobility Transistors (HEMTs)", NASA/TM-2003-211983, Available: http://gltrs.grc.nasa.gov/reports/ 2003/tm-2003-211983.pdf.

[12] S. M. Sze, Physics of Semiconductor Devices, 2nd ed., New York: Wiley, 1981.

[13] E. Etzkorn and D. Clarke, "Cracking of GaN films", J. Appl. Phys., vol. 89, 2001, pp. 1025. 